\title{
Characteristics of $\mathrm{HgCdTe}$ epilayer grown by LPE using horizontal slider
}

\author{
J K RADHAKRISHNAN*, S SITHARAMAN and S C GUPTA \\ Solid State Physics Laboratory, Lucknow Road, Delhi 110 054, India
}

\begin{abstract}
The characteristics of $\mathrm{HgCdTe}$ epilayers grown in a modified horizontal slider system, are reported here. The surface morphology of the grown layers, their IR transmission characteristics, depth and lateral compositional uniformity, structural and electrical characteristics are discussed.
\end{abstract}

Keywords. HgCdTe; LPE; characterization.

\section{Introduction}

Among the liquid phase epitaxial growth techniques, the one widely adapted for the growth of $\mathrm{HgCdTe}$ layer is the horizontal slider technique. The slider LPE growth from Te-rich solutions is used for growing $\mathrm{Hg}_{1-x} \mathrm{Cd}_{x} \mathrm{Te}$ epilayers, because of (i) the manageably small $\mathrm{Hg}$ partial pressure $(0 \cdot 1 \mathrm{~atm}$. at growth temperatures), the small melt volume requirements and the simplicity and economy of the technique, (ii) the ease of including additional in situ processes like substrate etching (melt-back), Hg-loss compensation, etc and (iii) the potential for growing heterostructures. This paper reports the characteristics of $\mathrm{HgCdTe}$ epilayers grown in a modified horizontal slider system.

\section{Experimental arrangement and $\mathrm{Hg}_{1-x} \mathrm{Cd}_{x} \mathrm{Te}$ epilayer growth}

The slider arrangement used here has provisions for (i) the compensation of $\mathrm{Hg}$ loss from growth solution, by providing an additional $\mathrm{Hg}$-vapour source in the form of HgTe kept inside the growth boat, (ii) an in situ meltback to remove the contaminated surface region of the substrate surface immediately prior to growth, (iii) an arrangement to push the layer/substrate intact out of the substrate-cavity immediately after growth and (iv) arrangements for reducing the volume of melt carry-over, when the substrate is removed from solution after growth ensuring a better wipe-off. $5 \mathrm{~g}$ of growth compound (molar fraction $\left(\mathrm{Hg}_{(1-z)} \mathrm{Cd}_{z}\right)_{(1-y)} \mathrm{Te}_{y}, z$ typically 0.049 and $y$ typically 0.84 ) is used to grow $\mathrm{Hg}_{1-x} \mathrm{Cd}_{x} \mathrm{Te}$ epilayers of area $10 \times 10 \mathrm{~mm}$ on $\mathrm{Cd}_{0.96} \mathrm{Zn}_{0.04} \mathrm{Te}$ substrates of area $15 \times$ $15 \mathrm{~mm}$ (Radhakrishnan et al 2001). The temperaturetime cycle for a typical epilayer growth is shown in

\footnotetext{
*Author for correspondence
}

figure 1 . In the present experiments the substrate was inserted under the growth solution well at a temperature $5^{\circ} \mathrm{C}$ above the liquidus temperature, to allow a sufficient meltback of the substrate surface and then reach equilibrium. The growth was performed for about $40 \mathrm{~min}$ by continuously ramping down the temperature. The layer was typically grown over a range $475^{\circ} \mathrm{C}-455^{\circ} \mathrm{C}$ at a cooling rate $0.5^{\circ} \mathrm{C} / \mathrm{min}$, giving a layer of $20-30 \mathrm{~m}$ thickness.

\section{Characteristics of $\mathrm{Hg}_{1-x} \mathrm{Cd}_{x} \mathrm{Te}$ epilayers}

The epilayer morphology shows terracing patterns and micropits/voids. Figure 2 shows some typical morphological features.

The IR transmission over an as-grown $\mathrm{HgCdTe}$ layer and the effect of thermal annealing at $200^{\circ} \mathrm{C}$ under $\mathrm{Hg}$ overpressure for $162 \mathrm{~h}$ are shown in figure 3. It is observed that, thermal annealing apart from improving the transmission of the layer, also gives rise to a sharper rise of the band-edge. Upon thermal annealing, point defects such as $\mathrm{Cd}$, Te precipitates which normally cause reduction in infrared transmission are annealed out and their size and density have been reduced considerably causing improved transmission. Also redistribution of constituents $\mathrm{Hg}, \mathrm{Cd}$ and $\mathrm{Te}$ takes place upon annealing improving the homogeneity of the epilayer giving rise to sharper rise of the band edge. The close spread of the band edge of the transmission curves of the annealed layer indicate a good lateral compositional uniformity. The Cd composition $(x)$ calculated from the IR transmission curves of the layer is $0 \cdot 22$.

Figure 4 shows the SEM picture and EDAX composition measurement across a cleaved edge of the layer. The SEM picture shows a $38 \mu$ thick $\mathrm{HgCdTe}$ epilayer and a planar substrate-epilayer interface. The depth composition analysis show a highly graded epilayer-interface region spreading over $3-4 \mu$. The rest of the layer has 
a nearly uniform $\mathrm{Cd}$ composition of around 0.22 ( 0.11 at.\%), a value similar to the one calculated from IR transmission. Over this region the $\mathrm{Cd}$ composition shows a small decrease towards the epilayer surface with a gradient of $9 \times 10^{-4} \mu \mathrm{m}^{-1}$.
The rocking curve over a portion of a $\mathrm{Hg}_{1-x} \mathrm{Cd}_{x} \mathrm{Te}$ epilayer, and the rocking curve on the $\mathrm{Cd}_{0.96} \mathrm{Zn}_{0.04} \mathrm{Te}$ substrate below it are shown in figure 5. The epilayer exhibits a FWHM of 93 arc s, which is very much lower than that of the substrate used. This kind of behaviour

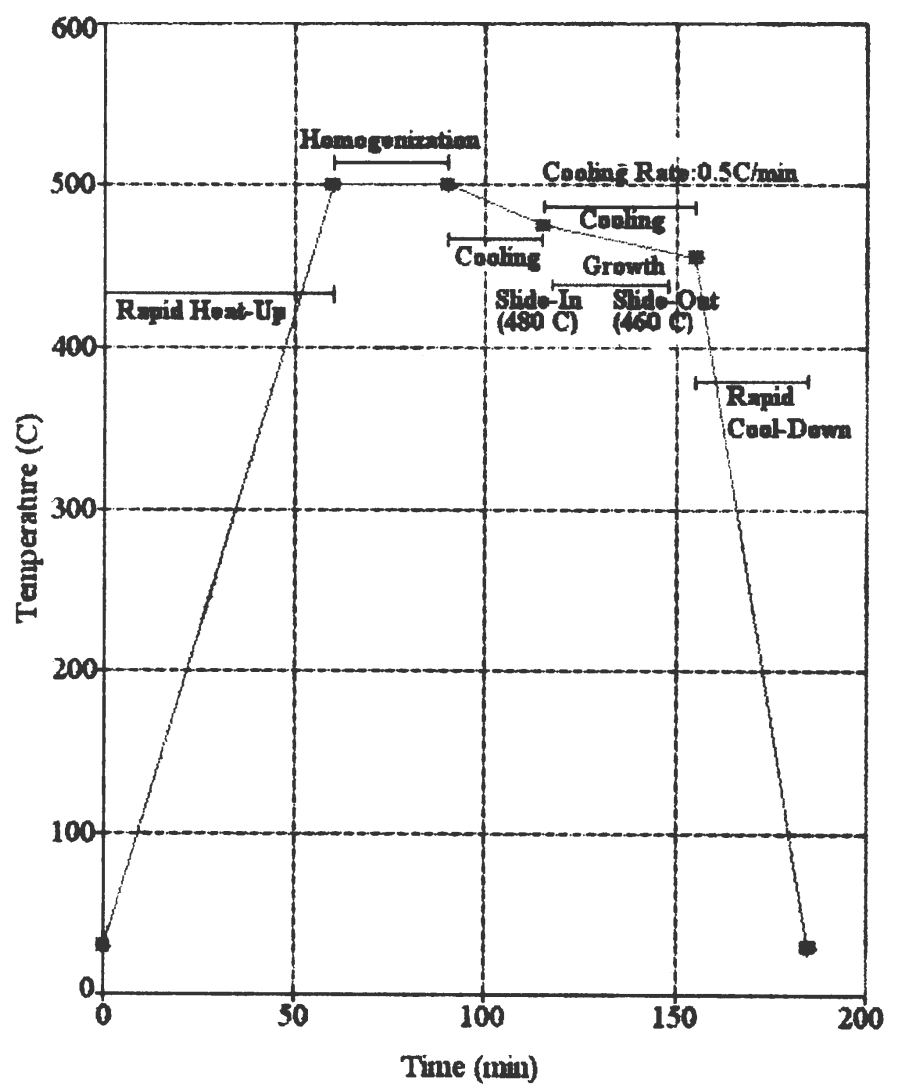

Figure 1. Temperature-time cycle for a typical epilayer growth.
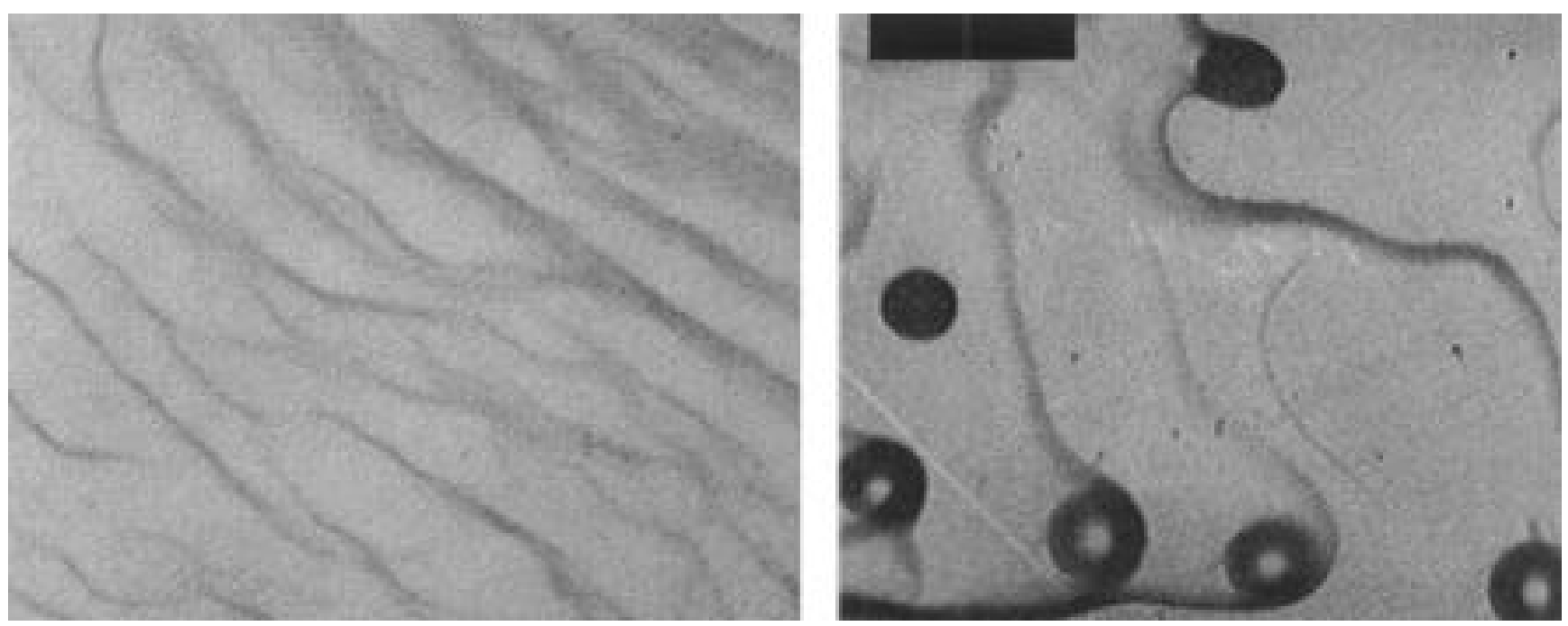

Figure 2. Some typical morphological features on $\mathrm{Hg}_{1-x} \mathrm{Cd}_{x} \mathrm{Te}$ layers grown using horizontal slider. 


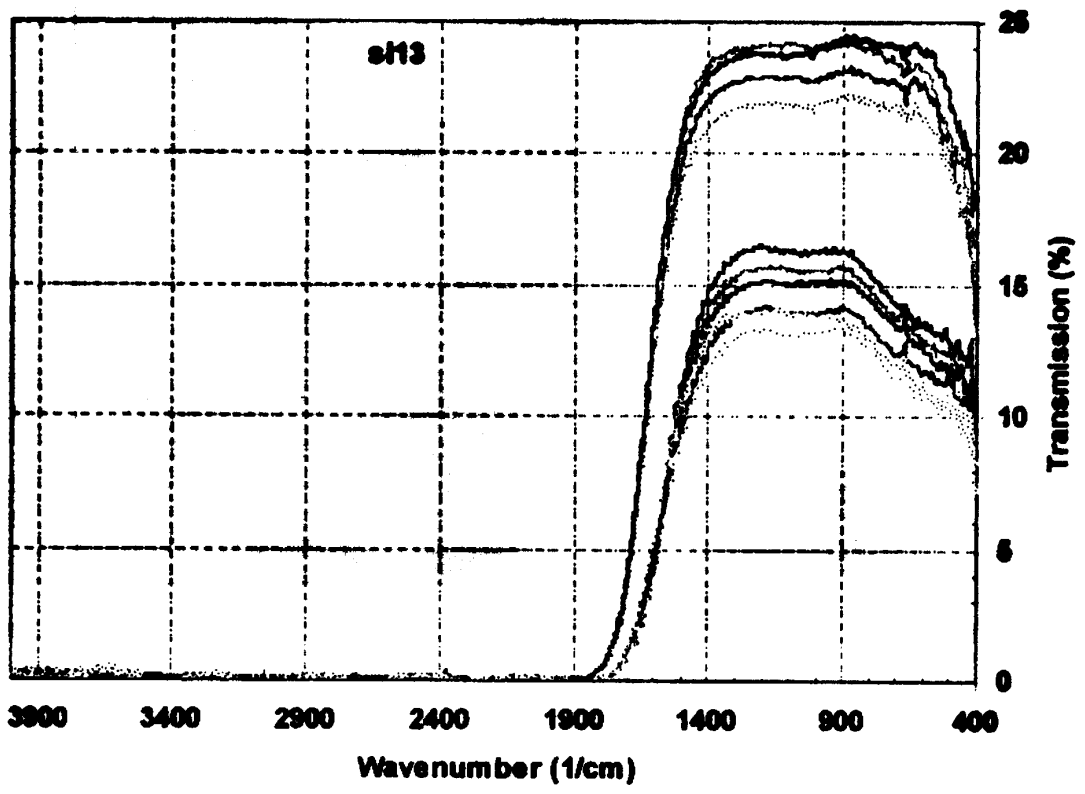

Figure 3. IR transmission over $\mathrm{Hg}_{1-x} \mathrm{Cd}_{x} \mathrm{Te}$ epilayer (SL-13-as grown and annealed).
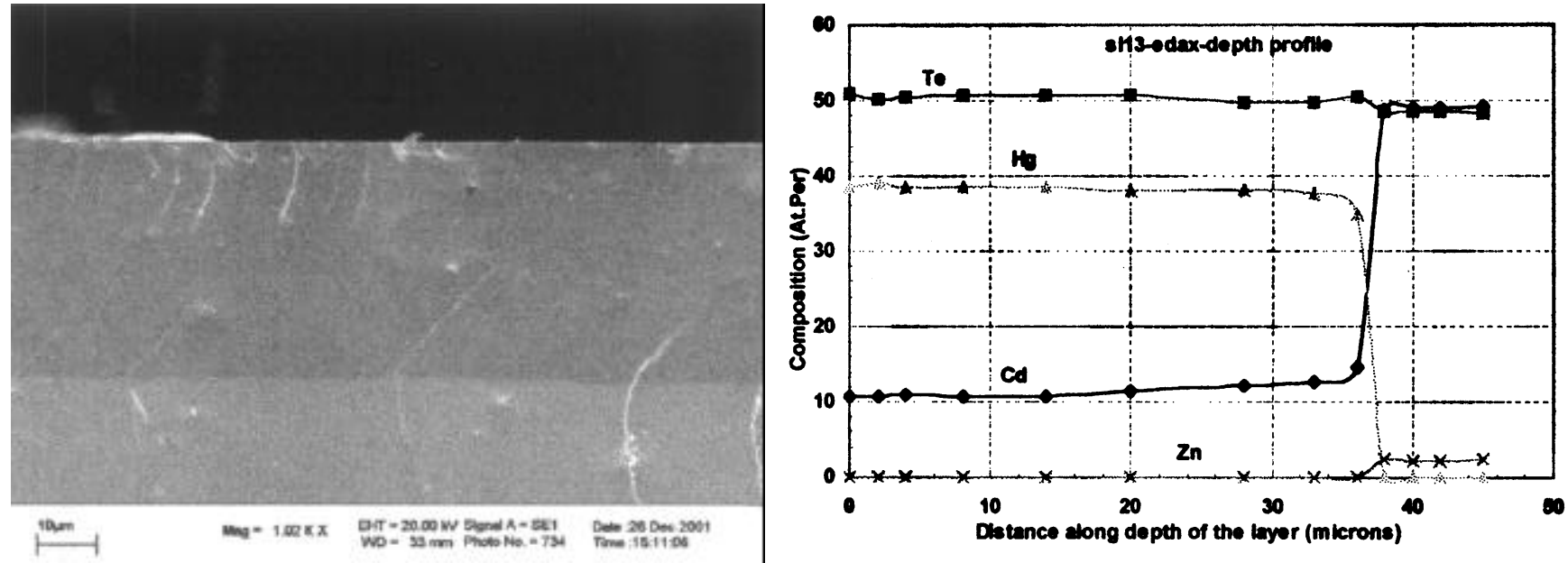

Figure 4. SEM picture and depth compositional measurement along the cleaved edge of a HgCdTe epilayer (SL-13).

has been observed in $\mathrm{HgCdTe} / \mathrm{CdZnTe}$, at high dislocation densities (and especially for thick films), where the dislocation density falls logarithmically over the first $5 \mu \mathrm{m}$ from the substrate-epilayer interface due to annihilation of threading dislocations, after which it remains approximately constant (Capper 1997).

The electrical properties of the layer at $77 \mathrm{~K}$ were measured by Van-der Pauw technique using a Bio-Rad system. Typical layers from initial experiments show the as grown layer (e.g. SL-13) to be $p$-type, with a carrier concentration of $1.4 \times 10^{17} \mathrm{~cm}^{-3}$ and a mobility of $260 \mathrm{~cm}^{2} \mathrm{~V}^{-1} \mathrm{~s}^{-1}$. Upon $n$-type annealing under $\mathrm{Hg}$ overpressure at $200^{\circ} \mathrm{C}$ for $262 \mathrm{~h}$, the annealed layer displays a carrier concentration of $9 \times 10^{16} \mathrm{~cm}^{-3}$ and a mobility of $11400 \mathrm{~cm}^{2} \mathrm{~V}^{-1} \mathrm{~s}^{-1}$. It is well known that the

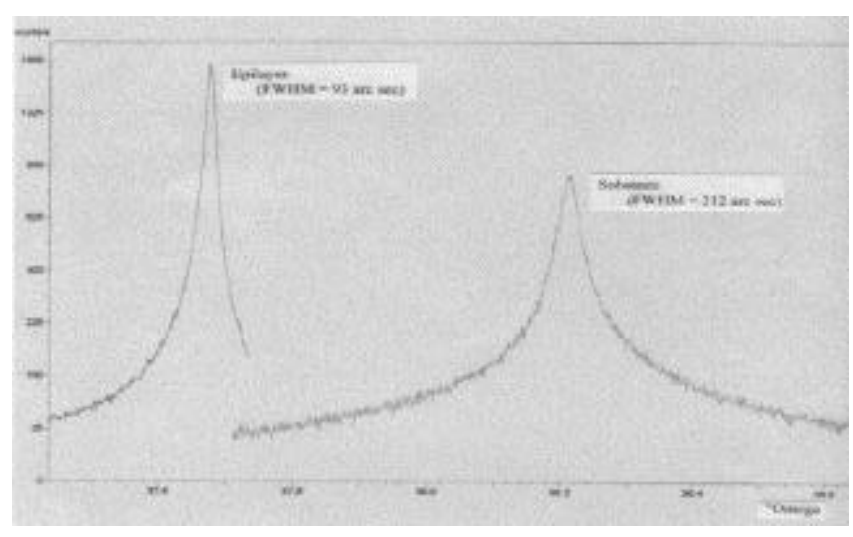

Figure 5. Rocking curve of the epilayer and the substrate (SL-13). 
electrical characteristics of the epitaxial layer is strongly influenced by the purity of the raw materials, the quality of the substrate and also show a dependence on the growth environment. Further, efforts are being made to improve the structural and electrical properties of the layer.

\section{Conclusion}

The results of the preliminary growth experiments of $\mathrm{HgCdTe}$ epilayer using a modified horizontal slider show smooth $\mathrm{HgCdTe}$ layers of $20-40 \mu$ thickness, with good lateral and depth compositional uniformity.

\section{Acknowledgements}

Thanks are due to Mr Vinod Kapoor for FTIR measurements, Mr Anand Kumar for EDAX measurements and Mr Anshu Goyal for rocking-curve measurements.

\section{References}

Capper P (ed.) 1997 Narrow gap II-VI compounds for optoelectronic and electromagnetic applications (UK: Chapman \& Hall) p. 357

Radhakrishnan J K, Durai L, Bhatnagar M K, Sitharaman S and Gupta S C 2001 Physics of semiconductor devices (eds) Vikram Kumar and P K Basu (New Delhi: Allied Publishers Ltd) p. 1182 